\title{
A hysteresis effect in the generation of field-aligned irregularities by a high-power radio wave
}

\author{
T. B. Jones and T. Robinson \\ Department of Physics, University of Leicester \\ P. Stubbe and H. Kopka \\ Max-Planck-Institut für Aeronomie
}

(Received September 15, 1982; accepted March 3, 1983.)

\begin{abstract}
The anomalous self-absorption of a high-power radio wave measured during recent ionospheric modification experiments exhibits a hysteresis effect. These results are consistent with the assumption that the growth of field-aligned irregularities due to the interaction of a high-power pump wave and the ionospheric plasma occurs in two stages. During the first stage, striation growth is due to an instability which has a threshold independent of striation amplitude. When the striation level is sufficiently high, the threshold of the second stage is reached. The second-stage threshold is dependent on the striation amplitude. The state of background ionosphere is also found to be an important factor influencing the observed effects.
\end{abstract}

\section{INTRODUCTION}

One of the most unexpected phenomena observed in the ionospheric modification experiments performed at Platteville, Colorado, was the generation of small-scale field-aligned irregularities (FAI) in the $F$ region just below the reflection height of a highpower radio wave [Fialer, 1974; Minkoff et al., 1974]. Only $O$ mode waves produced the striations, and it was therefore suggested that the excitation of Langmuir waves at the upper-hybrid frequency was an essential part of the generation mechanism, though the exact nature of the process is still not completely understood. The mechanisms proposed so far can be classified broadly into two types. Type 1 events have a power threshold for the high-power radio wave (pump) but require no initial striations to be present [Grach et al., 1977; Das and Fejer, 1979]. Type 2 have a threshold which depends on the product of pump power and the striation amplitude, producing explosive striation growth above the threshold [Vaskov and Gurevich, 1977; Inhester et al., 1981]. It has been suggested that the first mechanism may initially amplify striation amplitudes until the threshold of the second mechanism is reached, which then leads to further amplification of striation amplitudes

Copyright 1983 by the American Geophysical Union.

Paper number 3 S0379.

0048-6604/83/003S-0379\$08.00
[Inhester et al., 1981; Stubbe et al., 1982a]. As a result of the two-stage mechanism described above, it is expected that a hysteresis effect in the generation of striations by a pump wave should occur [Grach et al., 1978].

In a previous experiment at Platteville, Cohen and Whitehead [1970] demonstrated that an $O$ mode diagnostic wave was subject to anomalous absorption when propagating through a portion of the $F$ region heated by high-power radio waves. This phenomenon was subsequently explained in terms of the mode conversion of the em diagnostic wave into Langmuir waves because of coupling due to the plasma inhomogeneities associated with FAI generated by the pump [Graham and Fejer, 1976]. Anomalous absorption of the pump wave due to this mode conversion process plays an important role in the striation generation mechanism described above [Das and Fejer, 1979]. Theory suggests that anomalous absorption increases substantially for an em wave which propagates with its electric field nearly perpendicular to the geomagnetic field [Stubbe and Kopka, 1980]. Fieldaligned irregularities should therefore be more readily produced for vertically propagating em waves at high latitudes where the geomagnetic field is nearly vertical, resulting in greater anomalous absorption of em waves traversing the heated $F$ region. This was recently confirmed during cooperative experiments carried out jointly between the Max-Planck-Institut 

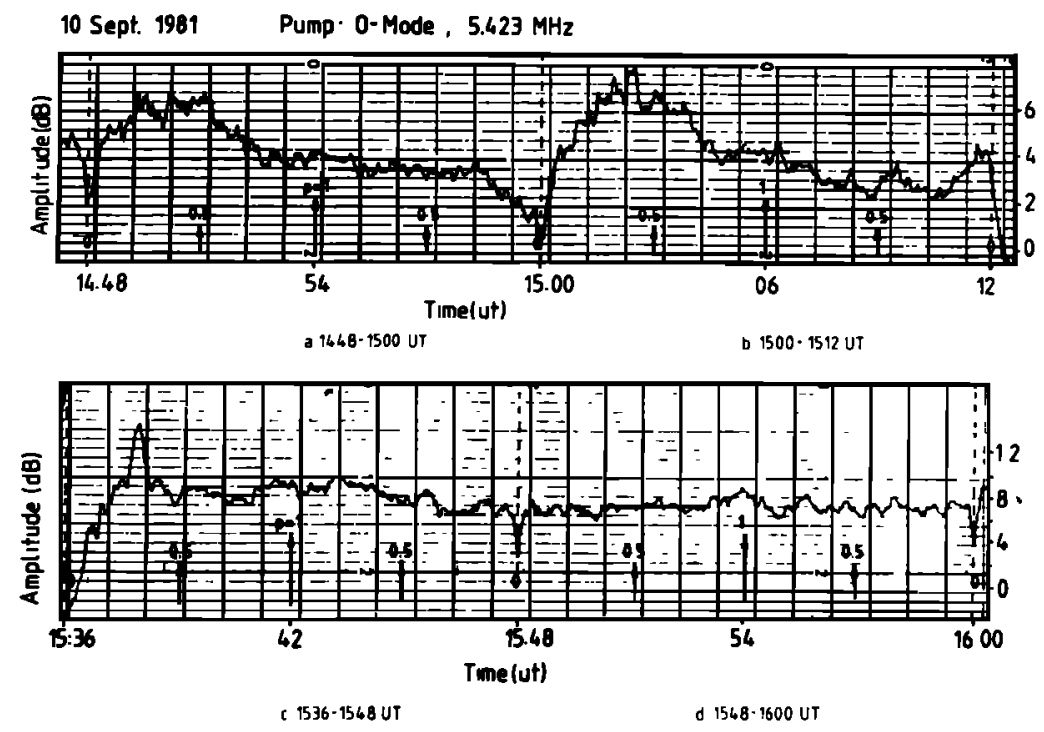

Fig. 1. Received signal strength of reflected pump wave during four periods of increasing and decreasing ERP, on September 10, 1981. The heater operated on $5.423 \mathrm{MHz}, O$ mode. During the first 6 min of each cycle, ERP was increased in forty $6.5-\mathrm{MW}$ steps from 0 to $260 \mathrm{MW}$ at 9-s intervals. During the next $6 \mathrm{~min}$, ERP was reduced in forty 6.5-MW steps from 260 to $0 \mathrm{MW}$.

(MPI) für Aeronomie, Lindau, and the University of Leicester using the new MPI high-power radio transmitter at Ramfjordmoen, Norway [Jones et al., 1982].

The essentially nonlinear nature of the anomalous absorption of high-power radio waves was demonstrated by Kopka et al. [1982] by measuring the amplitude of a pump wave after reflection from the ionosphere. These measurements revealed the existence of a hysteresis effect associated with the generation of field-aligned irregularities [Stubbe et al., $1982 b]$. In the following sections of this paper the hysteresis effect is discussed in the context of the mechanisms responsible for the generation of fieldaligned irregularities.

\section{PUMP WAVE MEASUREMENT}

During an experiment on September 10, 1981, the effective radiated power (ERP) of a 5.423-MHz pump wave was changed from 0 to $260 \mathrm{MW}$ (full power) by increasing the ERP in steps of $6.5 \mathrm{MW}$ at 9-s intervals. Subsequently, the ERP was progressively reduced by $6.5-\mathrm{MW}$ steps at 9-s intervals from full power down to $0 \mathrm{MW}$. Thus one complete cycle of increasing and decreasing ERP was completed in 12 min. This procedure was repeated several times. A receiver at a site some $40 \mathrm{~km}$ south of the pump transmitter measured the amplitude of the pump wave reflected from the ionosphere. The amplitude of two low-power diagnostic signals of 5.701 and 6.506 MHz from transmitters sited about $50 \mathrm{~km}$ north of the pump transmitter were also monitored at the same receiver site. The geometry of the diagnostic system ensured that the low-power waves passed through the heated $F$ region but not through those portions of the $D$ and $E$ regions illuminated by the pump. The diagnostic waves therefore monitored the anomalous absorption associated specifically with $F$ region phenomena.

In Figure 1 the received signal amplitudes for the 5.423-MHz pump are displayed for four consecutive cycles of increasing and decreasing ERP. There is a gap of $24 \mathrm{~min}$ between the end of cycle $b$ and the beginning of cycle $c$ during which time the pump was off. It is clear that cycles $a$ to $c$ are very similar. They exhibit the following common features: (1) a sudden rise in signal level during the first step from 0 to 6.5 MW ERP, (2) a slower rise during the subsequent steps up to about $30-50 \%$ of full power, (3) a fall in signal level during subsequent steps of increasing ERP, (4) a fairly constant level during the decrease from full power down to the last few steps before 0 MW is reached, and (5) a final rapid fall from 6.5 MW to $0 \mathrm{MW}$. Cycle $d$ does not exhibit features 2 and 3 . It consists of only feature 1 immediately followed by 4 and then 5 . 

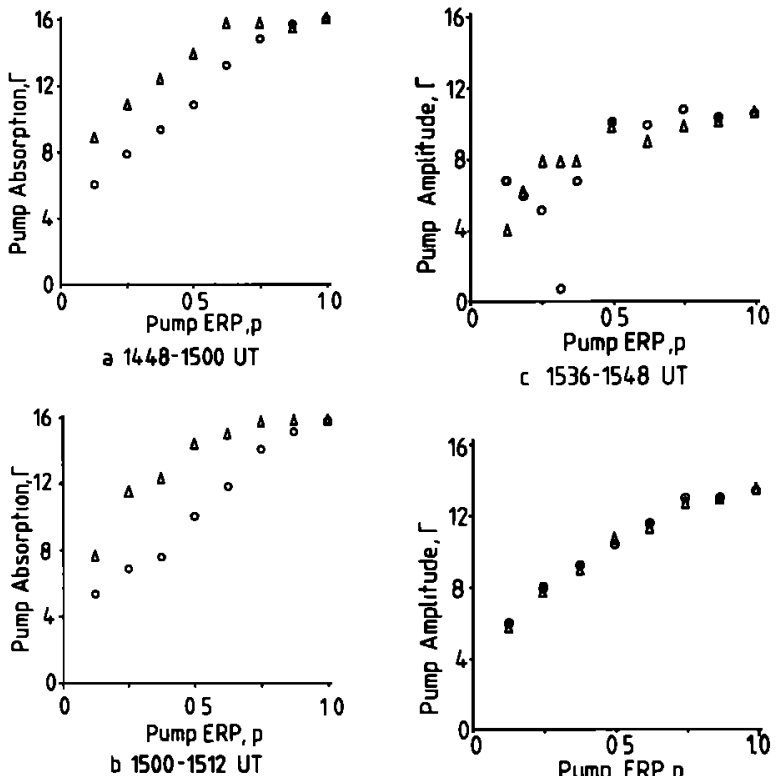

- increasing ERP a decreasing ERP

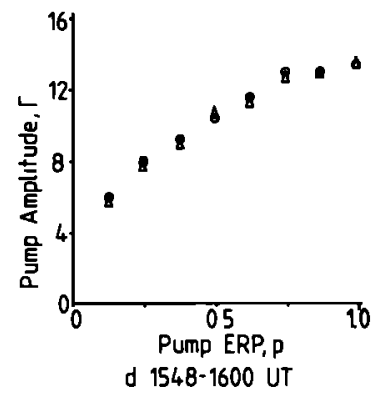

Fig. 2. Graphs of pump absorption, $\Gamma$ in decibels, against $\mathrm{ERP}=p \times 260 \mathrm{MW}(0 \leq p \leq 1)$ for each of the cycles in Figure 1 illustrating the difference $\Delta \Gamma$ (if any) between periods of increasing (circles) and decreasing (triangles) ERP.

An explanation of features 2 and 3 was given by Kopka et al. [1982]. That explanation is extended in the next section to include the asymmetry of the reflected pump amplitude between the stages of increasing and decreasing ERP exhibited in cycles $a$ to $c$, and to account for the reflected pump amplitude changes during cycle $d$.

\section{ANOMALOUS ABSORPTION: HYSTERESIS EFFECT}

If $P_{T}$ and $P_{R}$ are the ERP and received power levels of the pump, respectively, measured in decibels above an arbitrary level, an absorption index $\Gamma$ can be defined such that

$$
\Gamma=P_{T}-P_{R}
$$

In order to determine the effect of changing ERP on anomalous absorption due to field-aligned irregularities, $\Gamma$ was calculated for the data in Figure 1. For this purpose, $P_{T}$ was defined in decibels above 2.6 MW. No significance can be attributed to the absolute value of received signal strength because this very much depends on the receiver antenna configuration and location. Also, the value of $\Gamma$ does not represent the absolute absorption coefficient which must be defined in terms of the wave energy flux

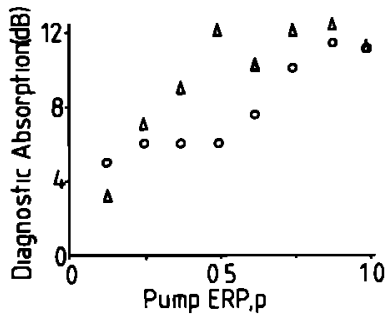

a. $5.701 \mathrm{MHz}, 1448-1500 \mathrm{UT}$

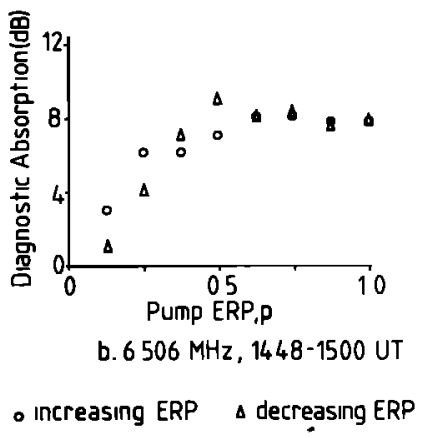

Fig. 3. Graphs of the absorption against pump ERP of two low-power diagnostic signals, (a) $5.701 \mathrm{MHz}$ and (b) $6.506 \mathrm{MHz}$ during cycle $a$ of Figure 1 .

entering and leaving a given region of the ionosphere. However, changes in $\Gamma$ with changing $P_{T}$ do measure the changes in nonlinear absorption. In the present case the $0-\mathrm{dB}$ level in the received signal represents a detected voltage of about $0.3 \mathrm{mV}$.

In Figure 2, graphs of $\Gamma$ as a function of fractional ERP have been plotted for each of the four cycles. Figure 3 illustrates the anomalous absorption of the 5.701- and 6.506- $\mathrm{MHz}$ diagnostics during cycle $a$. The diagnostic waves suffer anomalous absorption due to mode conversion into plasma waves in the heated $F$ region. Although a certain amount of nonlinear $D$ region absorption of the pump can be expected, the similarity between variations in the absorption levels of the diagnostic signals which respond only to $F$ region heating and that of the pump is evidence for the assumption that the variations in $\Gamma$ are due to anomalous absorptions of the pump itself in the $F$ region.

Figures $2 a-2 c$ illustrate clearly the existence of a hysteresis effect, although in the case of Figure $2 c$ it occurs only over a small ERP range corresponding to the sharp peak in the received pump signal which occurs at about 1538 UT. At a given value of ERP, $\Gamma$ has respective values $\Gamma_{i}$ and $\Gamma_{d}$ depending on whether the ERP is increasing or decreasing, such that

$$
\Gamma_{\mathrm{d}}-\Gamma_{i}=\Delta \Gamma>0
$$


The anomalous absorption of the 5.701-MHz diagnostic wave also exhibits hysteresis, though the effect does not appear to be present in the $6.506-\mathrm{MHz}$ diagnostic. An electron density profile constructed from ionograms taken at 1533 UT indicate that the reflection heights of the pump, the $5.701-\mathrm{MHz}$ and the 6.506- $\mathrm{MHz}$ diagnostics were, respectively, 248.4 $\mathrm{km}, 251.0 \mathrm{~km}$, and $262.0 \mathrm{~km}$. The difference in response of the two diagnostics can be explained in terms of the difference in the separations of their respective reflection points from that of the pump. This will be treated in detail in a later paper.

Kopka et al. [1982] have shown that in order for $P_{R}$ to decrease while ERP is still increasing, the condition

$$
P \partial \Gamma / \partial P>1
$$

must be satisfied, where $P$ is ERP measured in megawatts. It is evident that (3) can be satisfied if the gradient $\partial \Gamma / \partial P$ increases at a sufficiently large value of $P$. Figures $2 a-2 c$ all exhibit such an increase in $\partial \Gamma / \partial P$ at some critical value of $P\left(=P_{c}\right)$, at the point where $P_{R}$ maximizes. The increase in $\partial \Gamma / \partial P$ at $P=$ $P_{c}$ and the hysteresis effect which results when ERP is increased above $P_{c}$ are consistent with the onset of an instability of type 2 around $P=P_{c}$.

$\Delta \Gamma(P)$ generally falls off as $P-P_{c}$ increases. This is presumably because of saturation of the striation amplitude. The fact that $\Gamma$ was increasing with increasing ERP when $P<P_{c}$ indicates that striations had been generated at ERP values below $P_{c}$ probably by an earlier instability of type 1 whose threshold may well be exceeded during the first $6.5-\mathrm{MW}$ step.

In principle it is possible that the onset of the two types of instability, although temporally separated, may occur at the same ERP. This would be likely if the striation growth due to the type 1 instability were to have a high saturation amplitude so that the type 2 instability threshold was immediately exceeded. Stubbe et al. [1982a] have shown that this could be the case in a quiet ionosphere. Moreover, these authors found that in a time-varying ionosphere, the saturation level of the striation amplitude is greatly reduced and is highly power dependent. In this case a further rise in ERP may be required before the threshold of the type 2 instability was exceeded.

The absence of any noticeable hysteresis effect during cycle $d$ (Figure 1) suggests that the threshold of the type 2 instability was exceeded during the first step in ERP from 0 to $6.5 \mathrm{MW}$. The reduction in threshold power required could be a consequence of either changes in the variability of the ionosphere or of the influence of previous heating cycles in creating a residue of relatively large amplitude striations, amounting to a preconditioning of the ionosphere. Comparison of the ionogram taken during the experiment with those taken before and after indicated that the $F$ region critical frequency fell from $9.3 \mathrm{MHz}$ at $1408 \mathrm{UT}$ to $8.3 \mathrm{MHz}$ at $1533 \mathrm{UT}$ with a further drop to $6.5 \mathrm{MHz}$ by $1636 \mathrm{UT}$. It is concluded therefore that the changes observed in the variation of the received pump signal during the experiment were due to natural changes in ionosphere.

\section{CONCLUSIONS}

Results of a recent high-latitude modification experiment have been reported in which the signal strength of an ionospherically reflected high-power radio wave was measured while ERP from the highpower transmitter was steadily increased and then decreased. A hysteresis effect in the reflected pump amplitude was observed, i.e., at a given pump ERP; the reflected signal was smaller during the period of decreasing ERP than during the period of increasing ERP.

Changes in the effects observed during several repeated cycles of increasing and decreasing ERP can be attributed to natural changes in the background ionosphere as indicated by ionograms taken before, during, and after the experiment. This illustrates the important influence of the background ionospheric conditions on $F$ region modification.

The hysteresis effect is consistent with the excitation of field-aligned irregularities by the pump wave by the two-stage process described in the first section of this paper. However, further experimentation will be undertaken to verify this hypothesis. In these future experiments the anomalous absorption of both the pump and low-power diagnostic radio waves will be measured simultaneously with HF radar backscatter cross sections of the field-aligned irregularity.

\section{REFERENCES}

Cohen, R., and J. D. Whitehead, Radio-reflectivity detection of artificial modification of the ionospheric $F$ layer, $J$. Geophys. Res., 75, 6439, 1970.

Das, A. C., and J. A. Fejer, Resonance instability of small-scale field-aligned irregularities, J. Geophys. Res., 84, 6701, 1979.

Fialer, P. A., Field-aligned scattering from a heated region of the ionosphere: Observations at HF and VHF, Radio Sci., 9, 923, 1974. 
Grach, S. M., A. N. Karashtin, N. A. Mityakov, V. O. Rapoport, and V. Yu. Trakhtengerts, Parametric interaction of electromagnetic waves with ionospheric plasma, Radio Phys. Quantum Electron., Engl. Transl., 20, 1254, 1977.

Grach, S. M., A. N. Karashtin, N. A. Mityakov, V. O. Rapoport, and $\mathrm{V}$. Yu. Trakhtengerts, Thermal parametric instability in an inhomogeneous plasma (nonlinear theory), Sov. J. Plasma Phys., Engl. Transl., 4, 742, 1978.

Graham, K. N., and J. A. Fejer, Anomalous radio wave absorption due to ionospheric heating effects, Radio Sci., 11, 1057, 1976.

Inhester, B., A. C. Das, and J. A. Fejer, Generation of small-scale field-aligned irregularities in ionospheric heating experiments, $J$. Geophys. Res., 86, 9101, 1981.

Jones, T. B., T. Robinson, H. Kopka, and P. Stubbe, Phase changes induced in a diagnostic radio wave passing through a heated region of the auroral ionosphere, J. Geophys. Res., 87, $1557,1982$.

Kopka, H., P. Stubbe, T. B. Jones, and T. Robinson, Nonlinear reflectivity of high-power radio waves in the ionosphere, Nature, 295, 680, 1982.

Minkoff, J., P. Kugelman, and I. Weissman, Radio frequency scat- tering from a heated ionospheric volume, 1, VHF/UHF fieldaligned and plasma line backscatter measurements, Radio Sci., 9, 941, 1974.

Stubbe, $\mathrm{P}$., and H. Kopka, Modification of the $F$ region by powerful radio waves, in Exploration of the Polar Upper Atmosphere, edited by C. S. Deehr and J. A. Holtet, p. 83, D. Reidel, Hingham, Mass., 1980.

Stubbe, P., H. Kopka, T. B. Jones, and T. Robinson, Wide band attenuation of radio waves caused by powerful HF waves: Saturation and dependence on ionospheric variability, $J$. Geophys. Res., 87, 1551, $1982 a$.

Stubbe, $\mathbf{P}$., et al., Ionospheric modification experiments in northern Scandinavia, J. Atmos. Terr. Phys., 44, 1025, $1982 b$.

Vaskov, V. V., and A. V. Gurevich, Resonance instability of smallscale plasma perturbations, Sov. Phys. JETP, Engl. Transl., 46, $487,1977$.

T. B. Jones and T. Robinson, Department of Physics, University of Leicester, University Road, Leicester LE1 7RH, United Kingdom.

H. Kopka and P. Stubbe, Max-Planck-Institut für Aeronomie, 3411 Katlenburg-Lindau 3, Federal Republic of Germany. 